\title{
Basophil-lineage commitment in acute promyelocytic leukemia predicts for severe bleeding after starting therapy
}

\author{
Sergio Matarraz ${ }^{1,2} \cdot$ Pilar Leoz $^{1,2} \cdot$ Carlos Fernández $^{1,2} \cdot$ Enrique Colado $^{3} \cdot$ María Carmen Chillón $^{2,4}$. \\ María Belén Vidriales ${ }^{2,4} \cdot$ Marcos González $^{2,4} \cdot$ Daniel Rivera ${ }^{2,4} \cdot$ Carlos Salvador Osuna $^{5}$. \\ Teresa Caballero-Velázquez ${ }^{2,6} \cdot$ Vincent Van Der Velden $^{7} \cdot$ Mojca Jongen-Lavrencic ${ }^{8} \cdot$ Oliver Gutiérrez $^{9}$. \\ Ana Yeguas Bermejo ${ }^{10}$ - Luis García Alonso ${ }^{10}$ - Monique Bourgeois García ${ }^{11}$. Cristina De Ramón Sánchez ${ }^{11}$. \\ Gloria García-Donas ${ }^{12}$ - Aránzazu García Mateo ${ }^{13}$ - Isabel Recio ${ }^{14}$ - Javier Sánchez-Real ${ }^{15}$ - Andrea Mayado ${ }^{1,2}$. \\ María Laura Gutiérrez ${ }^{1,2}$. Paloma Bárcena ${ }^{1,2}$. Susana Barrena ${ }^{1,2}$. Antonio López ${ }^{1,2}$. Jacques Van Dongen ${ }^{16}$. \\ Alberto Orfao ${ }^{1,2}$
}

Received: 2 October 2017 / Revised: 24 January 2018 / Accepted: 26 January 2018 / Published online: 23 March 2018

(c) United States \& Canadian Academy of Pathology 2018

\begin{abstract}
Severe hemorrhagic events occur in a significant fraction of acute promyelocytic leukemia patients, either at presentation and/or early after starting therapy, leading to treatment failure and early deaths. However, identification of independent predictors for high-risk of severe bleeding at diagnosis, remains a challenge. Here, we investigated the immunophenotype of bone marrow leukemic cells from 109 newly diagnosed acute promyelocytic leukemia patients, particularly focusing on the identification of basophil-related features, and their potential association with severe bleeding episodes and patient overall survival.
\end{abstract}

From all phenotypes investigated on leukemic cells, expression of the CD203c and/or CD22 basophil-associated markers showed the strongest association with the occurrence and severity of bleeding $(p \leq 0.007)$; moreover, aberrant expression of $\mathrm{CD} 7$, coexpression of $\mathrm{CD} 34^{+} / \mathrm{CD}^{+}$and lack of $\mathrm{CD} 71$ was also more frequently found among patients with (mild and severe) bleeding at baseline and/or after starting treatment $(p \leq 0.009)$. Multivariate analysis showed that CD203c expression (hazard ratio: $26.4 ; p=0.003$ ) and older age (hazard ratio: $5.4 ; p=0.03$ ) were the best independent predictors for cumulative incidence of severe bleeding after starting therapy. In addition, CD203c expression on leukemic cells (hazard ratio: $4.4 ; p=0.01$ ), low fibrinogen levels (hazard ratio: $8.8 ; p=0.001$ ), older age (hazard ratio: $9.0 ; p=0.002$ ), and high leukocyte count (hazard ratio: $5.6 ; p=0.02$ ) were the most informative independent predictors for overall survival.

In summary, our results show that the presence of basophil-associated phenotypic characteristics on leukemic cells from acute promyelocytic leukemia patients at diagnosis is a powerful independent predictor for severe bleeding and overall survival, which might contribute in the future to (early) risk-adapted therapy decisions.

Electronic supplementary material The online version of this article (https://doi.org/10.1038/s41379-018-0038-2) contains supplementary material, which is available to authorized users.

Alberto Orfao

orfao@usal.es

Extended author information available on the last page of the article

\section{Introduction}

Acute promyelocytic leukemia is a unique subtype of acute myeloid leukemia with highly characteristic biological and clinical features associated with the rearrangement of the promyelocyte $(P M L)$ and retinoic acid receptor alpha (RARA) genes derived from $\mathrm{t}(15 ; 17)(\mathrm{q} 22 ; \mathrm{q} 11-12)$ [1]. Expression of the PML-RARA chimeric protein is usually associated with arrest of neutrophil differentiation at the promyelocyte stage [1]. Elucidation of the underlying molecular mechanisms of the disease has brought the first 
differentiation therapy for leukemia, based on administration of all-trans retinoic acid and/or arsenic trioxide, leading to high complete remission rates and cure of the disease in most (i.e., $\geq 90 \%$ ) patients [2].

Despite the high cure rates currently achieved with conventional promyelocytic leukemia-directed therapy, management of the disease still remains troublesome. This is mostly due to occurrence of life-threatening hemorrhagic episodes (and to a lesser extent also thrombotic events) translating into early deaths in 2.4 to $10 \%$ of patients, either before or immediately after starting therapy [3-5]. Altogether, this has fostered the search for additional parameters that would contribute to the identification of patients at higher risk of severe bleeding (and/or thrombosis), already at diagnosis. Thus, it is well-recognized that bleeding in promyelocytic leukemia is frequently associated with clinical and laboratory evidence of coagulopathy at disease onset $[6,7]$. These altered laboratory features have been related to the biological characteristics of leukemic promyelocytes [8], the presence of high white blood cell and peripheral blood blast cell counts (i.e., $>30 \times 10^{9} / \mathrm{L}$ ), an older patient age and abnormal creatinine serum levels [913]. In turn, hyperleukocytosis and/or increased blast cell counts are both typically linked to the microgranular (cytological) variant of the disease, while classical (hypergranular) promyelocytic leukemia patients most frequently present with pancytopenia; however, severe bleeding diathesis (at diagnosis and/or during treatment) is observed in both (cytological) disease subtypes [14]. Despite all the above associations, prediction of severe bleeding and early death of promyelocytic leukemia patients in clinical routine, still remains elusive.

Several studies have reported basophil-associated cytological and immunophenotypic features on leukemic cells from a subset of promyelocytic leukemia patients [15-17]. Interestingly, these unique tumor cell features have been frequently observed after administration of all-trans retinoic acid and/or arsenic trioxide [15-18]. At present, it is well recognized that normal human basophils contain and secrete a wide variety of mediators that include vasoactive (e.g., histamine) and anti-coagulant (e.g., heparin) compounds [19]. Whenever (massively) released, such compounds might induce severe hemostatic alterations [20]. Despite this, to the best of our knowledge no study has systematically investigated ex vivo the presence of immunophenotypic evidences of basophil differentiation on leukemic promyelocytes at disease diagnosis, and its potential association with severe bleeding and early death.

Here we performed an extensive immunophenotypic analysis of bone marrow leukemic cells from a cohort of 109 newly diagnosed and uniformly treated promyelocytic leukemia patients, focusing on the identification of basophil-associated maturation features on their leukemic cells, and the potential association with the occurrence and severity of bleeding episodes and early death.

\section{Methods}

\section{Patients and samples}

A total of 109 newly diagnosed and previously untreated acute promyelocytic leukemia patients ( $54 \%$ males and $46 \%$ females; median age of 54 years, ranging from 4 to 82 years) were investigated for the presence on bone marrow leukemic promyelocytes of phenotypes associated with basophil maturation. Diagnosis was based on morphological and immunophenotypic criteria, together with the presence of $P M L-R A R A$ gene rearrangements by interphase fluorescence in situ hybridization $(n=109)$ and molecular techniques $(n=76)$, following the World Health Organization 2008-2016 criteria [21].

In all 109 subjects investigated, complete blood cell counts and hemostatic parameters, including prothrombin time, (\%) prothrombin activity, international normalized ratio, partial thromboplastin time, (von Clauss) fibrinogen levels, D-dimer concentration, and antithrombin levels (\%), were assessed at diagnosis. Coagulopathy was defined as the presence of a prolonged prothrombin time $(>15 \mathrm{~s})$ or partial thromboplastin time ( $>40 \mathrm{~s}$ ), associated with hypofibrinogenemia $(<100 \mathrm{mg} / \mathrm{dL})$ and/or increased levels of fibrinogen degradation products (e.g., D-dimer $>500 \mathrm{ng} /$ $\mathrm{mL})$. In every patient, bleeding diathesis was documented at diagnosis $(n=109)$, and early during/after starting treatment $(n=106)$, being classified as: absent, mild (e.g., hematuria, petechia, ecchymosis, and/or hematomas) and severe bleeding (when transfusion of red cells was required due to severe metrorrhagia, intraabdominal hemorrhage, gastrointestinal tract bleeding and/or it affected vital organs, as in case of central nervous system bleeding and diffuse alveolar hemorrhage). According to the PETHEMA and GIMEMA criteria, $27 \%$ of patients were classified as lowrisk, $45 \%$ as intermediate-, and $28 \%$ as high-risk cases, based on both the leukocyte and platelet counts [10].

Patients were uniformly treated with the PETHEMA AIDA protocol that included induction therapy consisting of oral all-trans retinoic acid (i.e., $45 \mathrm{mg} / \mathrm{m}^{2}$ per day) divided into 2 daily doses, maintained until complete hematologic remission or for a maximum of 90 days, plus intravenous idarubicin (i.e., $12 \mathrm{mg} / \mathrm{m}^{2}$ per day) given on days $2,4,6$, and 8 , immediately after diagnosis.

\section{Cytomorphology}

Cytomorphological diagnosis was available for review in all patients; while $16 / 109$ cases $(15 \%)$ were diagnosed with a 
microgranular variant of promyelocytic leukemia, in the remaining 93 patients (85\%), leukemic cells showed the classical hypergranular promyelocytic appearance. The presence vs. absence of morphologic features associated/ suggesting basophil differentiation was also recorded.

\section{Immunophenotypic studies}

Flow cytometry immunophenotyping was performed at diagnosis on freshly obtained EDTA-anticoagulated bone marrow samples. Briefly, $50 \mu \mathrm{L}$ aliquots of whole bone marrow were stained with a standardized direct 8-color immunofluorescence stain-and-then-lyse technique, using the EuroFlow acute myeloid leukemia/myelodysplastic syndrome antibody panel [22]. Stained cells were measured in FACSCanto II flow cytometers equipped with the FACSDiva 6.1 software (Becton/Dickinson Biosciences, San Jose, California), except for 22 samples from one participating center that used a 4-color antibody panel that included an HLA-DR/CD203c/CD45/CD34 antibody combination analyzed in a Navios instrument (Beckman/Coulter, Miami, Florida). Flow cytometry listmode files were centrally and blindly re-analyzed at the Cytometry Service of the University of Salamanca (Salamanca, Spain) using the Infinicyt software (Cytognos SL, Salamanca, Spain). For all markers under study, a cut-off for positivity of $>2 \%$ was used. All bone marrow samples were systematically studied within the first $18 \mathrm{~h}$ after they had been drawn, after a written informed consent was provided by each subject, according to the guidelines of the local Ethics Committees and the Declaration of Helsinki.

\section{Detection of PML-RARA gene rearrangements and FLT3-ITD}

In every case, interphase fluorescence in situ hybridization aimed at detection of $\mathrm{t}(15 ; 17)$ and/or $\mathrm{t}(17 \mathrm{q} 12)$ chromosomal rearrangements was performed on interphase nuclei from whole bone marrow cells, after they were fixed in 3/1 (v/v) methanol/acetic. For this purpose, the following Spectrum Orange and Spectrum Green DNA probes (Vysis Inc., Downers Grove, Illinois) were used: LSI PML/RARA dualcolor, dual-fusion translocation probe and LSI RARA dualcolor breakapart rearrangement probe. Hybridization with fluorochrome-labeled probes was performed according to the recommendations of the manufacturer with slight modifications, as described elsewhere [23].

In addition, molecular analyses for both $P M L-R A R A$ gene rearrangements and FLT3-ITD were performed in mRNA samples. In brief, total RNA was isolated from whole bone marrow cells using Trizol (Invitrogen Carlsbad, California). Subsequently, mRNA was transcribed into cDNA according to the Europe Against Cancer Program protocols [24]. Absolute quantification of the different types of PML-RARA fusion transcripts was performed with such standardized protocols using a TaqMan-based real-time quantitative polymerase chain reaction and the ABL gene as an internal control [24]. FLT3-ITD was investigated by amplification of the juxtamembrane region spanning exons 14 and 15 , using a qualitative polymerase chain reaction (and fragment analysis) assay [25, 26].

\section{Statistical methods}

The SPSS software (IBM, Armonk, New York) was used for all statistical analyses. Multiple comparisons were performed using the $X^{2}$ test and either the Student's $t$ test (parametric data) or the Mann-Whitney $U$ and the Kruskal-Wallis tests (non-parametric data) for categorical and continuous variables, respectively. Receiver operating curves were used to establish cut-off values to predict for severe hemorrhage. Cumulative incidence of severe bleeding and overall survival curves were plotted using the Kaplan and Meier method; the log-rank test was used to establish statistical differences between survival curves.

\section{Results}

\section{Clinical and laboratory features of promyelocytic leukemia patients at diagnosis and their association with bleeding and overall survival}

From all 109 patients analyzed, $76(72 \%)$ showed signs of bleeding. Thus, mild bleeding was observed at diagnosis and during/after starting therapy in 55/109 (50\%) and 20/ 106 (19\%) patients, respectively, while severe bleeding occurred in 15/109 (14\%) and 22/106 (21\%) cases, respectively; 10 promyelocytic leukemia patients (9\%) exhibited severe bleeding both at diagnosis and after starting therapy, while 31 (29\%) showed no signs of bleeding, neither at diagnosis nor during follow-up.

Routine laboratory tests were altered in virtually all promyelocytic leukemia patients (Table 1). In detail, increased fibrin D-dimers, anemia, and thrombocytopenia were detected in virtually all subjects $(100 \%, 94 \%$, and $97 \%$ of cases, respectively), while leukopenia (58\%), increased prothrombin time $(57 \%)$ associated to a decreased prothrombin activity (53\%) and an abnormal international normalized ratio $(40 \%)$ were found in around half of the cases; altered creatinine serum levels, decreased fibrinogen and abnormal partial thromboplastin time were found at diagnosis in a smaller proportion of the patients $(29 \%, 17 \%$, and $11 \%$, respectively) (Table 1). Despite hemostatic tests were altered at diagnosis in the majority of patients (89\%), the frequency of such alterations was significantly higher 


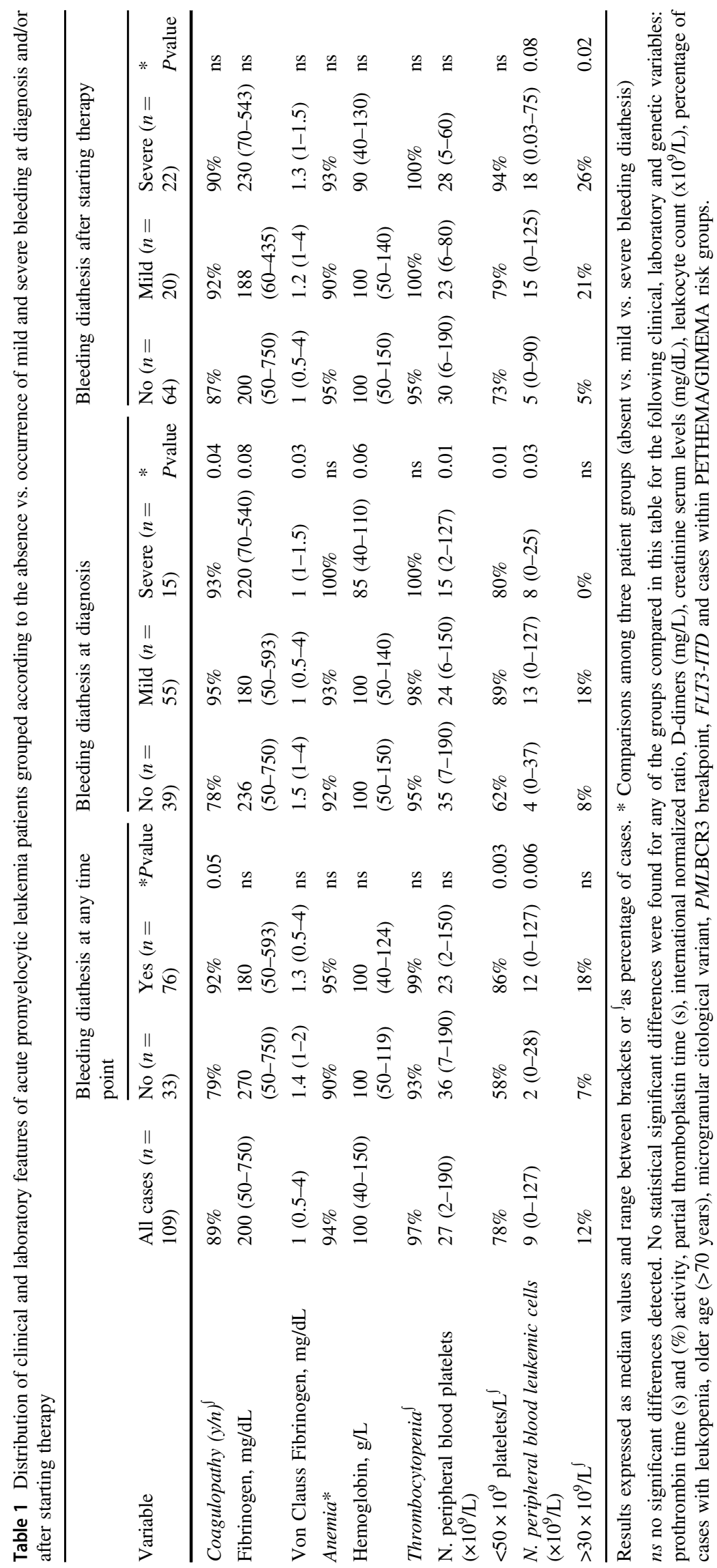


among patients that showed mild and severe signs of bleeding (95\% and 93\%, respectively) vs. those with no signs of hemorrhage at diagnosis ( $78 \%$ of cases; $p=0.04)$. Thus, lower fibrinogen levels (1.0 vs. $1.5 \mathrm{mg}$ von Clauss fibrinogen/dL; $p=0.03$ ), together with a higher frequency of severe thrombocytopenia $\left(<50 \times 10^{9}\right.$ platelets/L) were found in patients with mild and severe bleeding vs. those who did not show bleeding episodes $(89 \%$ and $80 \%$ vs. $62 \%$, respectively; $p=0.01$ ) (Table 1 ).

Moreover, $29 \%$ and $15 \%$ of promyelocytic leukemia patients had $>10$ and $>30$ white blood cells $\times 10^{9} / \mathrm{L}$, respectively, and $12 \%$ showed $>30 \times 10^{9} / \mathrm{L}$ leukemic cells in peripheral blood (Table 1). Of note, subjects within this later group more frequently showed (mild and severe) signs of bleeding after starting therapy vs. those who had lower leukemic cell counts: $39 \%$ and $39 \%$ vs. $21 \%$ and $15 \%$, respectively $(p=0.02)$ (Table 1$)$. In turn, $15 \%$ of the patients presented with the microgranular variant of the disease, while $85 \%$ had hypergranular leukemic promyelocytes associated with $B C R 1 / B C R 2$ or $B C R 3 P M L$ gene breakpoints in $68 \%$ and $32 \%$ of cases, respectively; FLT3ITD was found in $31 \%$ of patients. Leukemic promyelocytes from $76 \%$ of cases showed basophil maturation-associated cytological features (e.g., marked basophilic cytoplasm and/ or large basophilic granules). No significant association ( $p$ $>0.05$ ) was found between bleeding and the cytomorphological and molecular variants of the disease (Table 1).

Of note, a greater baseline percentage of bone marrow leukemic cells was observed among patients who showed mild and severe bleeding (vs. those who showed no bleeding), both before ( $85 \%$ and $84 \%$ vs. $74 \%$, respectively; $p=0.004)$ and after starting therapy (86\% and $86 \%$ vs. $80 \% ; p=0.01$ ) (Table 2). In turn, except for the percentage of residual $\mathrm{CD}_{3} 4^{+}$bone marrow hematopoietic progenitor and precursor cells, that was significantly lower among cases who presented with severe bleeding after starting therapy $(p=0.02)$, the distribution of all other residual bone marrow hematopoietic cell compartments was similar between individuals with and without bleeding diathesis (Supplementary Table 1).

\section{Immunophenotype of leukemic cells in promyelocytic leukemia and its association with bleeding diathesis}

Despite leukemic cells presented common immunophenotypic characteristics in virtually all promyelocytic leukemia cases (e.g., $\mathrm{CyMPO}^{+/++}, \mathrm{CD}^{\text {lo }}{ }^{\text {}}, \mathrm{CD} 117^{+/ \text {het }}, \mathrm{CD}^{+/++}$, $\mathrm{HLA}_{-D R^{-/ \mathrm{lo}}}, \mathrm{CD}_{3}{ }^{\text {hi }}, \mathrm{CD} 123^{+/ \mathrm{int}}, \mathrm{CD} 38^{+}, \mathrm{CD}^{-/ \mathrm{lo}}$, CD9

${ }^{+}, \mathrm{CD} 15^{-/ \mathrm{lo}}, \mathrm{CD}_{3} 6^{-}, \mathrm{CD} 14^{-}, \mathrm{CD} 300 \mathrm{e}^{-}, \mathrm{CD} 105^{-}, \mathrm{CD} 41^{-}$, $\mathrm{CD}^{-} 1^{-}, \mathrm{CD} 42 \mathrm{a}^{-}, \mathrm{CD} 42 \mathrm{~b}^{-}, \mathrm{CD} 10^{-}, \mathrm{CD} 16^{-}, \mathrm{CD} 25^{-}, \mathrm{CD} 3$ ${ }^{-}$, and $\mathrm{NG}^{-}$) (Fig. 1), variable antigen expression profiles were detected for several specific markers on leukemic promyelocytes from a subset of patients. Such variable phenotypes usually involved a subset of all leukemic cells, and included the expression of CD34 (>2\%-100\% of cells) in $49 \%$ of cases, asynchronous reactivity for CD35 $(>2 \%-60 \%)$ found in $38 \%$ of patients and presence of $\mathrm{CD} 4{ }^{\text {lo }}$ cells in one third of cases. In addition, a subset of the patients showed cross-lineage antigen expression consisting of positivity for CD7 (21\%), CD56 (20\%), CD2 (12\%), nuclear (Nu) TdT (9\%), CD19 (9\%), and CyCD79a (8\%) (Supplementary Table 2). Of note, reactivity for basophil/ mast cell-related markers such as CD203c and CD22 was also found in a variable proportion of leukemic promyelocytes from $30 \%$ and $12 \%$ of cases, respectively (Fig. 1 and Supplementary Table 2). Interestingly, most patients in whom their leukemic promyelocytes depicted phenotypic evidence of basophil-lineage maturation (i.e., CD203c $\mathrm{c}^{+}$ and/or CD22 $2^{+}$expression in the absence of $\left.\mathrm{CD} 117^{\mathrm{hi}}\right)$, more frequently showed immature features, as reflected by aberrant co-expression of CD34 (70\% vs. $40 \%$ of the other $\mathrm{CD} 203 \mathrm{c}^{-} \mathrm{CD}^{-} 2^{-}$cases; $\left.p=0.01\right)$.

From all phenotypic markers investigated (Supplementary Table 2), partial expression of the CD203c and/or CD22 basophil-associated antigens on bone marrow leukemic cells showed the strongest association with both the occurrence and the severity of bleeding at diagnosis and after starting therapy (Table 2). Thus, an increasing frequency of cases with $\mathrm{CD}_{203 c^{+}}$leukemic cells was observed among patients with severe hemorrhage vs. those with mild and no signs of bleeding, both at diagnosis $(87 \%$ vs. $17 \%$ and $6.5 \%$, respectively; $p<0.001)$ and after starting therapy ( $80 \%$ vs. $20 \%$ and $18 \%$, respectively; $p<$ $0.001)$. In detail, CD203c expression was detected in all $(100 \%)$ promyelocytic leukemia patients suffering from severe bleeding at diagnosis only (median: $20 \% \mathrm{CD}^{203 \mathrm{c}^{+}}$ bone marrow promyelocytes), in $90 \%$ of cases with severe hemorrhage both at diagnosis and during follow-up (median CD203c $\mathrm{c}^{+}$leukemic cells of $7 \%$ ) and in $73 \%$ of cases in whom severe bleeding emerged only after starting therapy (median: 4.0\% CD203c ${ }^{+}$tumor cells) $(p<0.001)$ (Table 2). In contrast, positivity for CD203c was present in only a minority of patients who showed no hemorrhage (6.5\% of cases) or displayed mild signs of bleeding (17\%) at diagnosis (median: $2.6 \%$ and $2.5 \% \mathrm{CD}^{203 \mathrm{c}^{+}}$bone marrow promyelocytes, respectively; $p<0.001$ ), and in $6 \%$ of cases with no signs of bleeding at any time point (median of $2 \%$ CD203c ${ }^{+}$bone marrow promyelocytes; $p<$ 0.001). Interestingly, higher relative and absolute numbers of peripheral blood $\mathrm{CD}_{2} 03 \mathrm{c}^{+}$leukemic cells were also detected in patients with severe bleeding vs. those with mild or no signs of bleeding, both at diagnosis-median of $4 \%$ vs. $1 \%$ and $0.5 \%$ peripheral blood CD203c ${ }^{+}$leukemic cells $(p<0.001)$, and of 0.5 vs. 0.2 and 0.2 CD203c ${ }^{+}$ leukemic cells $\times 10^{9} / \mathrm{L}(p<0.001)$, respectively-and after 


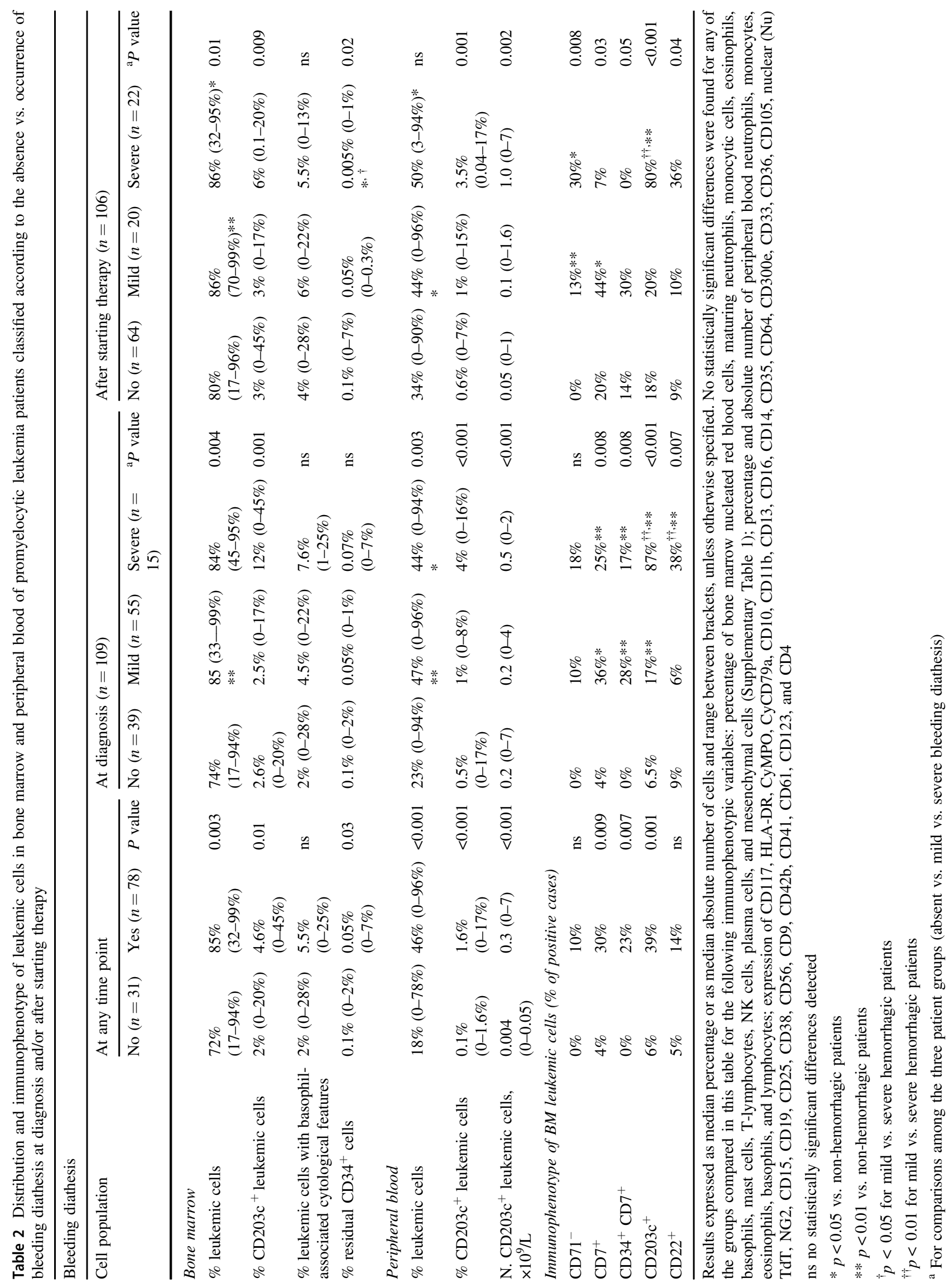


Fig. 1 Immunophenotypic characterization of bone marrow cells from a patient with promyelocytic leukemia. a Automatic population separator plot allowing ten-dimensional discrimination of two populations of promyelocytic leukemic cells (light blue and red dots) from neutrophillineage cells (dark blue), CD34 + precursors (green) and other residual hematopoietic cells (gray events). The minor subset of leukemic cells (highlighted red) can be distinguished from the major leukemic cell population based on their differential expression of the CD $34^{+}$vs. CD $34^{-}, \mathrm{CD} 45^{\mathrm{hi}}$ vs. $\mathrm{CD} 45^{\text {lo }}(\mathbf{b}, \mathbf{c}), \mathrm{CD} 11 \mathrm{~b}^{+}$vs. CD11b ${ }^{-}$, CD64 $^{\text {lo }}$ vs. CD64 $4^{\text {hi }}$ (g, h), $\mathrm{CD} 33^{\text {hi }}$ vs. CD $33^{\text {int }}$, and CD 4 ${ }^{+}$vs. $\mathrm{CD} 4^{-}$markers $(\mathbf{j}, \mathbf{k})$ and the expression of the basophil maturation-associated markers CD203c, CD22 and CD123 ${ }^{\text {hi }}$ (m-o). In turn, all leukemic cells from both populations similarly showed variable positivity for CD117, $\mathrm{CyMPO}^{+}$, and CD38 ${ }^{+}$ (d-f), a CD $15^{-/ l o}$ phenotype (i), together with positivity for CD71 (l)
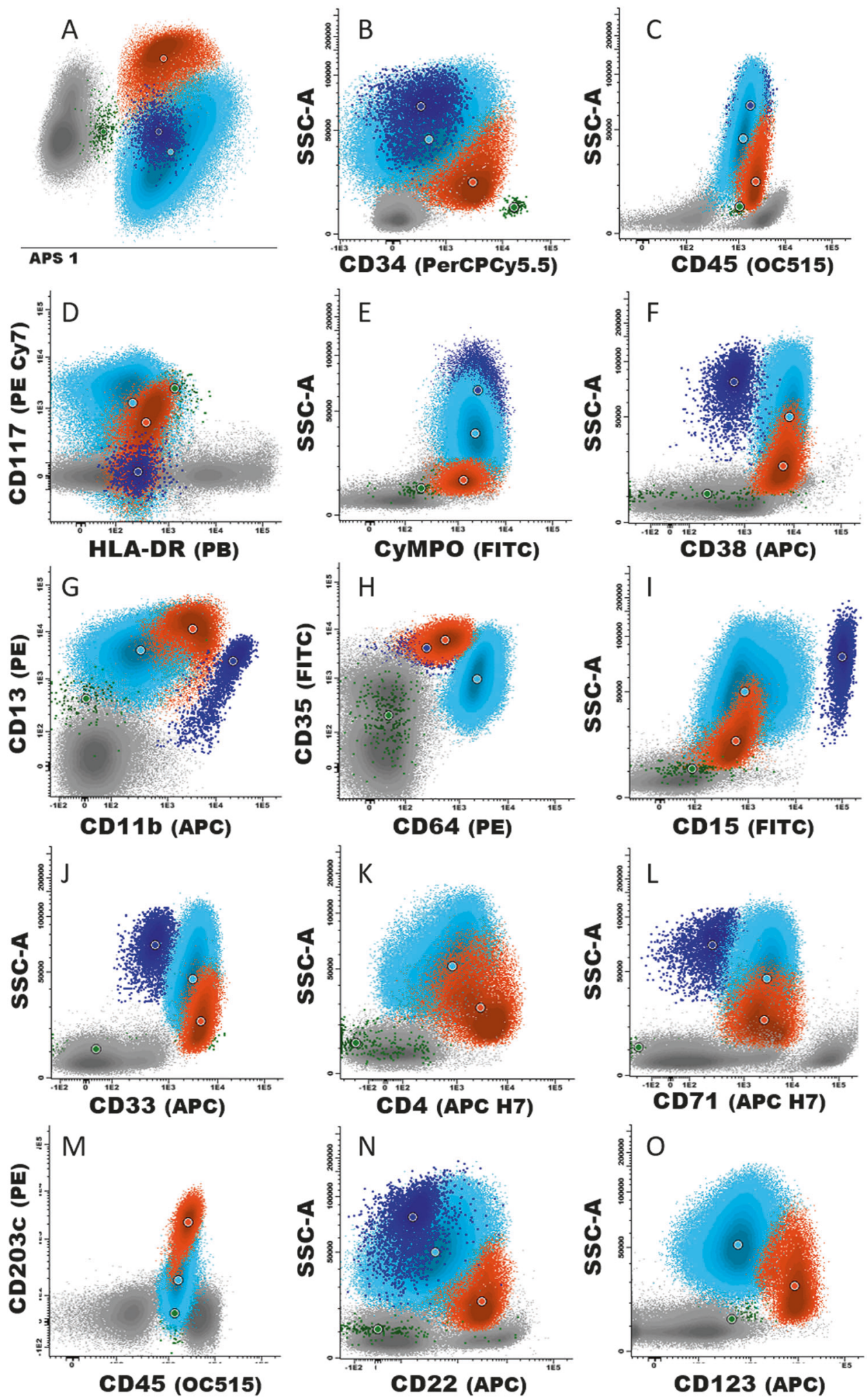

starting therapy-median of 1.0 vs. 0.1 and 0.05 cells $\times 10^{9} / \mathrm{L}(p=0.002)$ - (Table 2$)$.

Similarly, CD22 ${ }^{+}$leukemic cells were more frequently found among subjects with severe bleeding vs. those with mild and no signs of bleeding at diagnosis $(38 \%$ vs. $6 \%$ and $9 \%$, respectively; $p=0.007)$ and after starting therapy (36\% vs. $10 \%$ and $9 \% ; p=0.04$ ) (Table 2). In addition, the percentage of cases with aberrant CD7 expression and a CD34 
${ }^{+} \mathrm{CD}^{+}$phenotype on leukemic cells, was also higher among individuals showing (mild and severe) hemorrhage vs. those who had no signs of bleeding, at any time point evaluated (30\% and $23 \%$ vs. $4 \%$ and $0 \%$, respectively; $p \leq$ 0.009) (Table 2). In turn, lack of CD71 expression on bone marrow leukemic promyelocytes was also more commonly observed among patients with mild and severe bleeding after starting therapy, while CD71 was systematically positive in cases that had no signs of hemorrhage $(\%$ of CD71-negative cases of $13 \%$ and $30 \%$ vs. $0 \%$, respectively; $p=0.008$ ) (Table 2). No significant association was found between bleeding after starting therapy and the expression of any of the other immunophenotypic markers evaluated $(p>0.05)$.

Interestingly, $92 \%$ of cases presenting with CD203c ${ }^{+}$ leukemic promyelocytes also showed cytological features associated with basophil maturation, while this occurred in $66 \%$ of the CD203c ${ }^{-}$cases $(p=0.04)$.

\section{Prognostic impact of the immunophenotype of leukemic promyelocytes and other disease features on the cumulative incidence of severe bleeding and patient overall survival}

Promyelocytic leukemia patients in whom leukemic cells showed expression of the CD203c $\left(>2 \% \mathrm{CD}_{2} 03 \mathrm{c}^{+}\right.$bone marrow leukemic cells and $>0.2 \%$ peripheral blood CD203c leukemic cells; $p<0.001)$ and CD22 $(p=0.005)$ basophil-associated markers, as well as those cases presenting with high leukocyte and leukemia cell counts $\left(>30 \times 10^{9} / \mathrm{L} ; p \leq 0.001\right)$ and age $>70$ years $(p=0.005)$, showed a significantly higher overall cumulative incidence of severe bleeding (Table 3 and Fig. 2). Except for a high leukocyte count, all the above parameters were also associated with higher cumulative incidence of severe bleeding after starting therapy ( $p \leq 0.05$; Table 3 and Fig. 2). This translated into a significantly shorter overall survival for patients $>70$ years $(p=0.01)$, cases showing CD203c ${ }^{+}(p$ $=0.007)$, and $\mathrm{CD} 6^{+} \mathrm{CD} 7^{+}$bone marrow leukemic cells $(p$ $=0.04)$, high peripheral blood leukocyte $\left(>30 \times 10^{9} / \mathrm{L} ; p \leq\right.$ $0.03)$ and leukemic cell counts $\left(>30 \times 10^{9} / \mathrm{L} ; p=0.05\right)$, together with decreased fibrinogen levels $(<100 \mathrm{mg} / \mathrm{dL} ; p=$ $0.01)$, an altered international normalized ratio $(>1.3 ; p=$ $0.02)$ and low platelet counts $\left(<50 \times 10^{9}\right.$ platelets $/ \mathrm{L} ; p=$ 0.04 ) at diagnosis (Table 3 and Fig. 3).

Multivariate analysis of prognostic factors showed that high peripheral blood leukemic cell counts $\left(>30 \times 10^{9} / \mathrm{L}\right)$ was the only parameter showing an independent predictive value for severe bleeding at any time point evaluated (hazard ratio: $22.4 ; 95 \% \mathrm{CI}: 2.3-216 ; p=0.007$ ), while CD203c expression on leukemic promyelocytes (hazard ratio: $26.4 ; 95 \% \mathrm{CI}: 3-224 ; p=0.003)$ together with an older age (hazard ratio: $5.4 ; 95 \%$ CI: $1-25 ; p=0.03$ ) was the best combination of prognostic factors for cumulative incidence of severe bleeding after starting therapy. In turn, low fibrinogen levels (hazard ratio: 8.8 ; 95\% CI: $2.5-31 ; p$ $=0.001$ ), age $>70$ years (hazard ratio: $9.0 ; 95 \%$ CI: $2-37 ; p$ $=0.002)$, high $\left(>30 \times 10^{9} / \mathrm{L}\right)$ leukocyte count (hazard ratio: 5.6; 95\% CI: $1.3-24 ; p=0.02$ ), and CD203c expression on bone marrow leukemic cells (hazard ratio: 4.4; 95\% CI: $1.3-14 ; p=0.01)$ were the most informative independent predictors for overall survival of acute promyelocytic leukemia patients (Table 3).

\section{Discussion}

In recent decades, treatment of promyelocytic leukemia has significantly advanced, achieving $>90 \%$ cure rates [27, 28]. Despite the management of bleeding complications occurring in these patients has also significantly improved with the introduction of all-trans retinoic acid therapy to the standard care of the disease [8], a significant fraction of patients suffer from severe bleeding already at presentation and/or immediately after starting therapy, leading to an increased incidence of treatment failure and early mortality, worldwide [3, 5-7, 14, 27, 29-33]. Early hemorrhagic deaths among promyelocytic leukemia patients has long been associated with administration of cytotoxic chemotherapy, owing to destruction of leukemic cells $[29,30$, 34]. Such leukemic cell damage may trigger complex coagulopathy processes, promoting the enhancement of a usually pre-existing pro-coagulant and hyperfibrinolytic environment, mediated by the (sudden) massive exposure to tumor cell-derived components released by the dying leukemic promyelocytes, such as tissue factor, cancer procoagulant and annexin-II [35]. This, together with an enhanced non-specific protease activity and cytokine production (e.g., IL-1 $\beta, \mathrm{TNF} \alpha$ ), also associated with tumor cells and tumor cell death, leads to a greater consumption of coagulation factors and circulating platelets, further increasing the risk of bleeding and thrombotic events in these patients [11, 13].

Although increasing knowledge exists about the coagulopathy that occurs in promyelocytic leukemia patients [1, 14, 32, 36], the specific mechanisms involved still need to be precisely defined. Furthermore, current prognostic factors predicting for severe hemorrhage, particularly after initiating therapy (e.g., high leukocyte and leukemia cell counts) remain of limited utility, particularly for the most prevalent (often leukopenic) hypergranular disease subtype $[3,6,9,37]$. Thereby, identification of additional predictive factors is required for optimal prognostication and better management of bleeding in these patients.

In promyelocytic leukemia, detection of metachromatic basophilic granulation in the cytoplasm of leukemic 


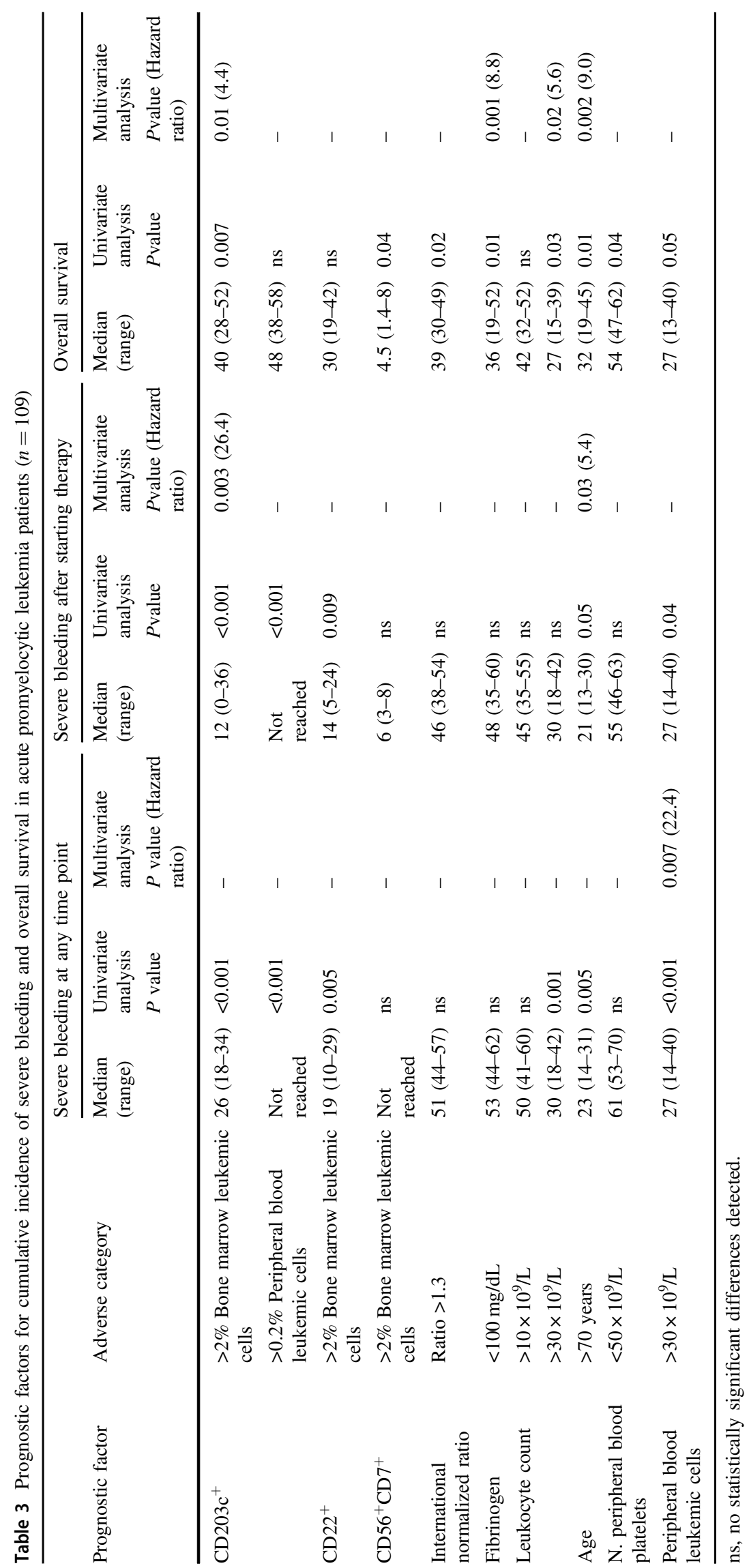




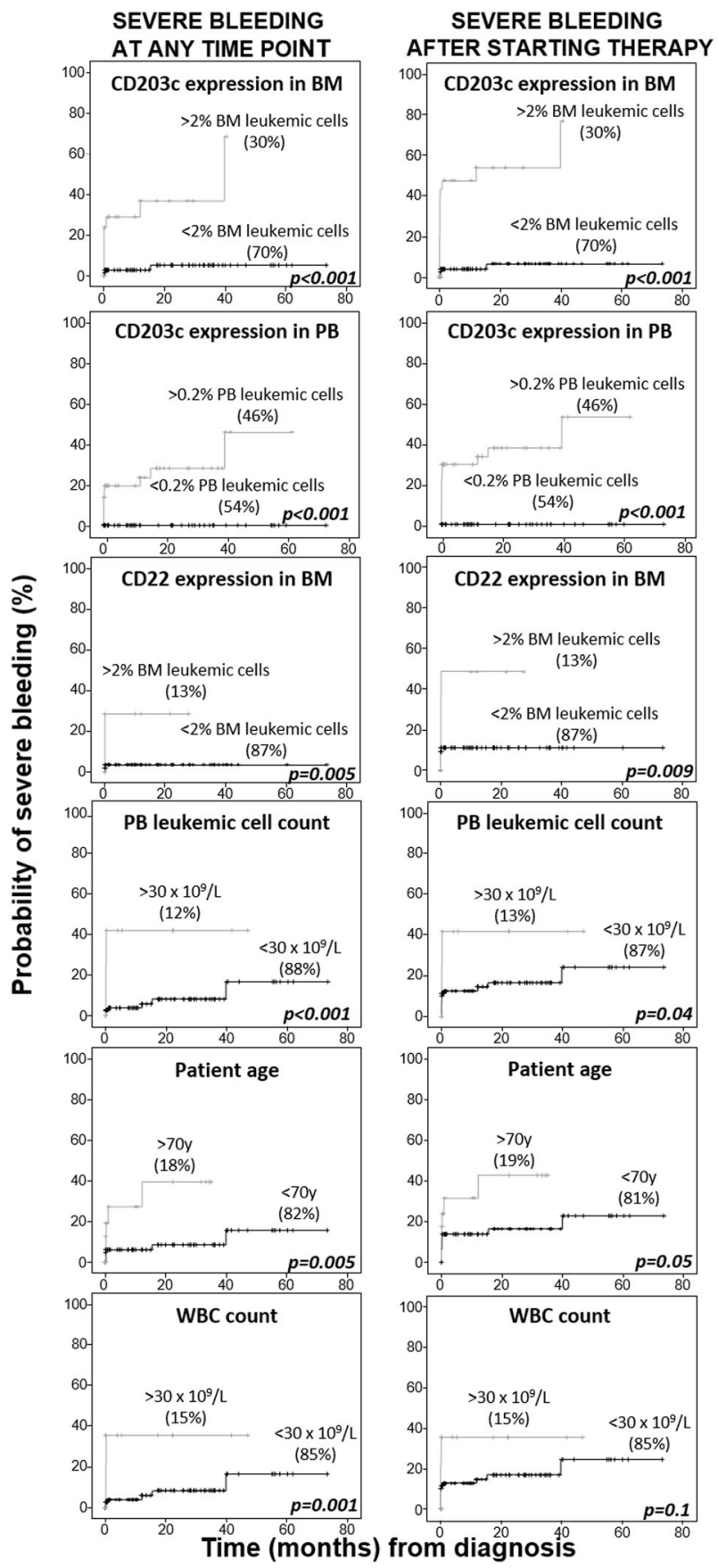

Fig. 2 Impact of the expression of basophil-associated immunophenotypic markers on leukemic cells and other disease features on cumulative incidence of severe bleeding occurring in promyelocytic leukemia at any time (left panels) or only after therapy had started (right panels) $(n=109)$

promyelocytes, together with the development of hyperhistaminemia, after starting therapy [15, 38-40], have long suggested that basophil-associated vasoactive mediators (e.g., heparin, histamine) might be released by those leukemic promyelocytes upon spontaneous or chemotherapyinduced cell differentiation and/or death; thereby, such release of basophil-related contents by these leukemic cells might contribute, at least in part, to the development of an anticoagulant state and a higher risk for severe bleeding. In line with this hypothesis, maturation of leukemic promyelocytes toward the basophil lineage has been recurrently reported in the literature, particularly after all-trans retinoic acid therapy [15-18].

Based on an extensive and detailed immunophenotypic analysis, here we confirm that leukemic promyelocytes typically display features associated with neutrophilcommitment $\left(\mathrm{CyMPO}^{+}\right)$, and a maturation blockade around the promyelocyte stage (HLA-DR ${ }^{-/ l o}, \mathrm{CD} 33^{\text {hi }}$, $\mathrm{CD} 117^{\mathrm{lo} /+}$ ), together with aberrantly low expression of $\mathrm{CD} 15$ and variable reactivity for CD34, CD71, CD4, CD7, CD35, CD56, and CD64, among other markers; in addition, expression of $\mathrm{CD}_{123^{+ \text {int }}}, \mathrm{CD} 8^{+}$, and $\mathrm{CD}^{+}$was also found on leukemic promyelocytes from virtually all cases studied at diagnosis, fully in line with previous observations [23, 41, 42]. Most interestingly, expression of basophilassociated markers such as CD203c and/ or CD22, involving a subset of leukemic promyelocytes, was found in up to one third of patients who also showed more frequently cytomorphological features associated with basophil maturation. Of note, unequivocal positivity for these markers (CD203c and/or CD22) on $\geq 2 \%$ bone marrow leukemic cells, together with aberrant CD7 expression, lack of CD71 and co-expression of both CD7 and CD34, were all significantly associated with the occurrence of bleeding in our promyelocytic leukemia patients. However, from these markers, only the CD203c and CD22 basophil-associated proteins showed a significant impact on the cumulative incidence of severe bleeding in these patients. In detail, $\mathrm{CD} 203 \mathrm{c}$ expression in a fraction of both bone marrow and peripheral blood leukemic cells was found in the vast majority of cases who showed severe hemorrhage at diagnosis and after starting therapy, while it was absent in virtually all subjects without bleeding episodes.

Within hematopoietic cells, CD203c expression-i.e., ecto-nucleotide pyrophosphatase/phosphodiesterase-3 (ENPP3) - has been specifically associated with basophil and mast cell differentiation, from very early to late maturation stages [43-45]. Among these cell lineages, most CD203c ${ }^{+}$ cells also coexpress CD22 and other basophil/mast cellassociated markers (e.g., tryptase, chymase), with either a $\mathrm{CD} 123^{+} / \mathrm{CD} 117^{-/ \mathrm{lo}}$ basophil-related or a CD117 ${ }^{\text {hi }} \mathrm{CD} 123$ -/lo mast cell-associated phenotype [43]. Hence, immunophenotyping provides high accuracy for detection of leukemic promyelocytes with basophil-lineage features. As mentioned above, massive death of leukemic cells after starting therapy might contribute to explain the increased frequency of bleeding among this subgroup of promyelocytic leukemia patients presenting with $\mathrm{CD} 203 \mathrm{c}^{+}$and/or $\mathrm{CD} 22^{+}$expression. Of note, positivity for $\mathrm{CD} 203 \mathrm{c}$ has also been previously reported in (several) other tumor cell types 
Fig. 3 Impact of the immunophenotype of leukemic cells and other clinical and laboratory disease features on overall survival of promyelocytic leukemia patients $(n=109)$
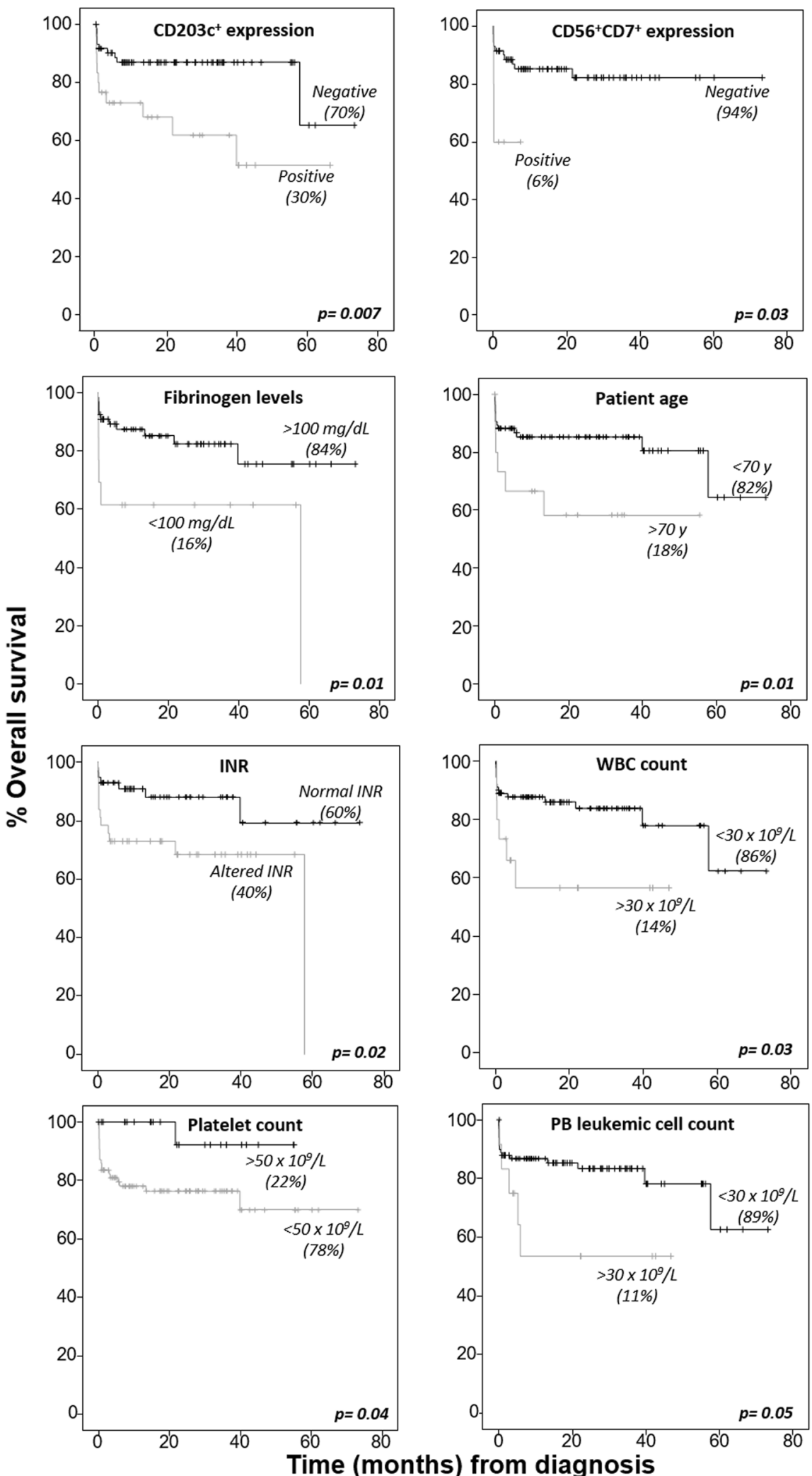

including breast cancer, colon adenocarcinoma, neoplastic bile duct carcinoma and glioma, where it has been associated with a greater tumor cell motility, migration and tissue invasion [46-48]; consequently, expression of CD203c could also potentially confer an enhanced tissue invasive capacity on leukemic promyelocytes (e.g., to the 
central nervous system), thereby also promoting development of local (e.g., intracranial) severe tissue bleeding. In this regard, expression of molecules of the NPP family, such as CD203c, have also been inversely associated with the plasma levels of angiotensin II and platelet-derived growth factor [49]; despite this, only a statistical nonsignificant tendency toward a higher frequency of severe thrombocytopenia $\left(<50 \times 10^{9}\right.$ platelets/L) was found when we compared cases who presented with vs. without CD203c expression on their leukemic cells ( $87 \%$ vs. $74 \%$ of cases; $p$ $>0.05$ ), suggesting a marginal role for CD203c expression on the pathogenesis of thrombocytopenia in these patients.

Hemorrhagic syndrome in promyelocytic leukemia has been frequently associated with high-risk disease features, such as leukocytosis $\left(>10 \times 10^{9} / \mathrm{L}\right)$ [50]. In addition, highrisk patients have been reported to display specific leukemia cell phenotypes (e.g., expression of CD34, CD2, CD7, CD15, and CD56), molecular alterations (e.g., FLT3-ITD and/or the $B C R 3 P M L$ breakpoint), and a microgranular leukemic cell morphology, in association with an unfavorable outcome [51, 52]. From all such adverse disease features, high leukocyte counts and older age have been recurrently associated with an increased risk of bleeding [3, $6,9,37,53]$, as also confirmed here. As also found by other groups [3, 6, 9, 37, 53], our results further showed an association between both the overall number of (bone marrow and peripheral blood) leukemic cells and the presence of hemostatic alterations (including low fibrinogen levels and platelet counts), and the occurrence of bleeding episodes; however, no significant differences were observed for these parameters between subjects with distinct degree (mild vs. severe) of bleeding symptoms [3, 6, 9, 37, 53]. In contrast, the presence of basophil maturation-associated phenotypic features on leukemic promyelocytes, emerged as a strong and independent predictor for both severe bleeding (early) after starting therapy and overall patient survival, particularly when combined with advanced age alone or together with other previously identified prognostic factors (altered fibrinogen, international normalized ratio and high leukocyte count), respectively.

In summary, here we show for the first time that basophil maturation of leukemic promyelocytes defined on immunophenotypic grounds occurs in around one third of promyelocytic leukemia patients, providing a greater risk of severe bleeding both at diagnosis and (particularly) after starting therapy. Thus, expression of the CD203c basophil maturation-associated marker on leukemic cells showed a strong and independent predictive value for both the occurrence of severe bleeding after starting therapy and overall survival of these patients. Further studies in larger series of promyelocytic leukemia patients are required to confirm our findings, and to establish preventive and/or treatment measures for an improved management of patients at increased risk for severe bleeding, particularly among this unique subgroup of $\mathrm{CD} 203 \mathrm{c}^{+}$promyelocytic leukemia cases.

Acknowledgements This work was supported by the Fundación Científica de la Asociación Española Contra el Cáncer (AECC, Madrid, Spain) and the Fundación Rafael del Pino (Madrid, Spain) and both CIBERONC (CB16/12/00400, CB16/12/00233, CB16/12/00480) and grant PI16/00787 from Instituto de Salud Carlos III (Ministerio de Economía y Competitividad, Madrid, Spain).

Author contributions SM performed research, analyzed the data and wrote the manuscript; CF and PL contributed substantially to the laboratory work and analyzed data; EC provided patient samples and clinical data and analyzed data; MCC and MG performed molecular studies; CSO, TC-V, VVDV, MJ-L, OG, AYB, LGA, GG-D, DR, MBG and CDRS provided patient samples and retrieved relevant clinical information from patient records; AM critically revised the paper and analyzed data; MLG performed iFISH studies; PB performed FACS-sorting studies; MBV and AL compiled immunophenotypic data files and reports; JVD provided patient samples and clinical data; AO designed research, analyzed the data and wrote the manuscript. All authors have contributed to the writing review and approved the manuscript.

\section{Compliance with ethical standards}

Conflict of interest The authors declare that they have no conflict of interest.

\section{References}

1. Lo-Coco F, Hasan SK. Understanding the molecular pathogenesis of acute promyelocytic leukemia. Best Pract Res Clin Haematol. 2014;27:3-9.

2. Lo-Coco F, Di Donato L, Gimema, et al. Targeted therapy alone for acute promyelocytic leukemia. N Engl J Med. 2016;374: 1197-8.

3. de la Serna J, Montesinos P, Vellenga E, et al. Causes and prognostic factors of remission induction failure in patients with acute promyelocytic leukemia treated with all-trans retinoic acid and idarubicin. Blood. 2008;111:3395-402.

4. Lehmann S, Ravn A, Carlsson L, et al. Continuing high early death rate in acute promyelocytic leukemia: a population-based report from the Swedish Adult Acute. Leuk Regist Leuk. 2011;25:1128-34.

5. Abla O, Ribeiro RC, Testi AM, et al. Predictors of thrombohemorrhagic early death in children and adolescents with $t(15 ; 17)$ positive acute promyelocytic leukemia treated with ATRA and chemotherapy. Ann Hematol. 2017;96:1449-56.

6. Kim DY, Lee JH, Lee JH, et al. Significance of fibrinogen, Ddimer, and LDH levels in predicting the risk of bleeding in patients with acute promyelocytic leukemia. Leuk Res. 2011;35:152-8.

7. Chang H, Kuo MC, Shih LY, et al. Clinical bleeding events and laboratory coagulation profiles in acute promyelocytic leukemia. Eur J Haematol. 2012;88:321-8.

8. Falanga A, Rickles FR. Pathogenesis and management of the bleeding diathesis in acute promyelocytic leukaemia. Best Pract Res Clin Haematol. 2003;16:463-82.

9. Dally N, Hoffman R, Haddad N, et al. Predictive factors of bleeding and thrombosis during induction therapy in acute promyelocytic leukemia-a single center experience in 34 patients. Thromb Res. 2005;116:109-14.

10. Sanz MA, Lo Coco F, Martin G, et al. Definition of relapse risk and role of nonanthracycline drugs for consolidation in patients 
with acute promyelocytic leukemia: a joint study of the PETHEMA and GIMEMA cooperative groups. Blood. 2000;96:1247-53.

11. Tallman M, Lo-Coco F, Kwaan H, Sanz M, Gore S. Clinical roundtable monograph. Early death in patients with acute promyelocytic leukemia. Clin Adv Hematol Oncol. 2011;9:1-16.

12. Tallman MS, Lo-Coco F, Kwaan HC, Sanz MA, Gore SD. Early death in patients with acute promyelocytic leukemia. Proceedings from a live roundtable at the 2010 American Society of Hematology Annual Meeting, December 4-7, 2010, Orlando, Florida. Clin Adv Hematol Oncol. 2011;9:1-16.

13. Park JH, Qiao B, Panageas KS, et al. Early death rate in acute promyelocytic leukemia remains high despite all-trans retinoic acid. Blood. 2011;118:1248-54.

14. Breen KA, Grimwade D, Hunt BJ. The pathogenesis and management of the coagulopathy of acute promyelocytic leukaemia. Br J Haematol. 2012;156:24-36.

15. Masamoto Y, Nannya Y, Arai S, et al. Evidence for basophilic differentiation of acute promyelocytic leukaemia cells during arsenic trioxide therapy. Br J Haematol. 2009;144:798-9.

16. Iwakiri R, Inokuchi K, Dan K, Nomura T. Marked basophilia in acute promyelocytic leukaemia treated with all-trans retinoic acid: molecular analysis of the cell origin of the basophils. Br J Haematol. 1994;86:870-2.

17. Tallman MS, Hakimian D, Snower D, et al. Basophilic differentiation in acute promyelocytic leukemia. Leukemia. 1993;7: 521-6.

18. Kubonishi I, Fujishita M, Niiya K, et al. Basophilic differentiation in acute promyelocytic leukemia. Nihon Ketsueki Gakkai Zasshi. 1985;48:1390-6.

19. Holgate ST. The role of mast cells and basophils in inflammation. Clin Exp Allergy. 2000;30(Suppl 1):28-32.

20. Siracusa MC, Perrigoue JG, Comeau MR, Artis D. New paradigms in basophil development, regulation and function. Immunol Cell Biol. 2010;88:275-84.

21. Arber DA, Orazi A, Hasserjian R, et al. The 2016 revision to the World Health Organization classification of myeloid neoplasms and acute leukemia. Blood. 2016;127:2391-405.

22. van Dongen JJ, Lhermitte L, Bottcher S, et al. EuroFlow antibody panels for standardized n-dimensional flow cytometric immunophenotyping of normal, reactive and malignant leukocytes. Leukemia. 2012;26:1908-75.

23. Orfao A, Chillon MC, Bortoluci AM, et al. The flow cytometric pattern of CD34, CD15 and CD13 expression in acute myeloblastic leukemia is highly characteristic of the presence of PMLRARalpha gene rearrangements. Haematologica. 1999;84: 405-12.

24. Gabert J, Beillard E, van der Velden VH, et al. Standardization and quality control studies of 'real-time' quantitative reverse transcriptase polymerase chain reaction of fusion gene transcripts for residual disease detection in leukemia-a Europe Against Cancer program. Leukemia. 2003;17:2318-57.

25. Chillon MC, Fernandez C, Garcia-Sanz R, et al. FLT3-activating mutations are associated with poor prognostic features in AML at diagnosis but they are not an independent prognostic factor. Hematol J. 2004;5:239-46.

26. Thiede C, Steudel C, Mohr B, et al. Analysis of FLT3-activating mutations in 979 patients with acute myelogenous leukemia: association with FAB subtypes and identification of subgroups with poor prognosis. Blood. 2002;99:4326-35.

27. Watts JM, Tallman MS. Acute promyelocytic leukemia: what is the new standard of care? Blood Rev. 2014;28:205-12.

28. Cicconi L, Lo-Coco F. Current management of newly diagnosed acute promyelocytic leukemia. Ann Oncol. 2016;27:1474-81.

29. Altman JK, Rademaker A, Cull E, et al. Administration of ATRA to newly diagnosed patients with acute promyelocytic leukemia is delayed contributing to early hemorrhagic death. Leuk Res. 2013;37:1004-9.

30. Breccia M, Lo Coco F. Thrombo-hemorrhagic deaths in acute promyelocytic leukemia. Thromb Res. 2014;133(Suppl 2): S112-116.

31. Choudhry A, DeLoughery TG. Bleeding and thrombosis in acute promyelocytic leukemia. Am J Hematol. 2012;87:596-603.

32. Mantha S, Tallman MS, Soff GA. What's new in the pathogenesis of the coagulopathy in acute promyelocytic leukemia? Curr Opin Hematol. 2016;23:121-6.

33. Rahme R, Thomas X, Recher C, et al. Early death in acute promyelocytic leukemia (APL) in French centers: a multicenter study in 399 patients. Leukemia. 2014;28:2422-4.

34. Sanz MA, Montesinos P. How we prevent and treat differentiation syndrome in patients with acute promyelocytic leukemia. Blood. 2014;123:2777-82.

35. Wang ZY, Chen Z. Acute promyelocytic leukemia: from highly fatal to highly curable. Blood. 2008;111:2505-15.

36. Kwaan HC, Cull EH. The coagulopathy in acute promyelocytic leukaemia--what have we learned in the past twenty years. Best Pract Res Clin Haematol. 2014;27:11-18.

37. Yanada M, Matsushita T, Asou N, et al. Severe hemorrhagic complications during remission induction therapy for acute promyelocytic leukemia: incidence, risk factors, and influence on outcome. Eur J Haematol. 2007;78:213-9.

38. Shimamoto Y, Suga K, Yamaguchi M, Kuriyama K, Tomonaga M. Prophylaxis of symptoms of hyperhistaminemia after the treatment of acute promyelocytic leukemia with all-trans retinoic acid. Acta Haematol. 1994;92:109-12.

39. Koike T, Tatewaki W, Aoki A, et al. Brief report: severe symptoms of hyperhistaminemia after the treatment of acute promyelocytic leukemia with tretinoin (all-trans-retinoic acid). N Engl J Med. 1992;327:385-7.

40. Castoldi GL, Liso V, Specchia G, Tomasi P. Acute promyelocytic leukemia: morphological aspects. Leukemia. 1994;8: 1441-6.

41. Chen Z, Li Y, Tong Y, et al. Stepwise discriminant function analysis for rapid identification of acute promyelocytic leukemia from acute myeloid leukemia with multiparameter flow cytometry. Int J Hematol. 2016;103:306-15.

42. Dong HY, Kung JX, Bhardwaj V, McGill J. Flow cytometry rapidly identifies all acute promyelocytic leukemias with high specificity independent of underlying cytogenetic abnormalities. Am J Clin Pathol. 2011;135:76-84.

43. Teodosio C, Mayado A, Sanchez-Munoz L, et al. The immunophenotype of mast cells and its utility in the diagnostic work-up of systemic mastocytosis. J Leukoc Biol. 2015;97:49-59.

44. Han X, Jorgensen JL, Brahmandam A, et al. Immunophenotypic study of basophils by multiparameter flow cytometry. Arch Pathol Lab Med. 2008;132:813-9.

45. Falcone FH, Haas H, Gibbs BF. The human basophil: a new appreciation of its role in immune responses. Blood. 2000;96:4028-38.

46. Yano Y, Hayashi Y, Sano K, et al. Expression and localization of ecto-nucleotide pyrophosphatase/phosphodiesterase I-3 (E-NPP3/ CD203c/PD-I beta/B10/gp130RB13-6) in human colon carcinoma. Int J Mol Med. 2003;12:763-6.

47. Yano Y, Hayashi Y, Sano K, et al. Expression and localization of ecto-nucleotide pyrophosphatase/phosphodiesterase I-1 (E-NPP1/ PC-1) and -3 (E-NPP3/CD203c/PD-Ibeta/B10/gp130(RB13-6)) in inflammatory and neoplastic bile duct diseases. Cancer Lett. 2004;207:139-47.

48. Deissler H, Blass-Kampmann S, Bruyneel E, Mareel M, Rajewsky MF. Neural cell surface differentiation antigengp130(RB13-6) induces fibroblasts and glioma cells to express astroglial proteins and invasive properties. FASEB J. 1999;13:657-66. 
49. Kettenhofen R, Meyer zu Brickwedde MK, Ko Y, Vetter H, Sachinidis A. Identification of a phosphodiesterase I/nucleotide pyrophosphatase-related gene mRNA in rat vascular smooth muscle cells by the differential display approach. J Mol Biol. 1998;279:323-9.

50. Lo-Coco F, Cicconi L. What is the standard regimen for patients with acute promyelocytic leukemia? Curr Hematol Malig Rep. 2014;9:138-43.

51. Testa U, Lo-Coco F. Prognostic factors in acute promyelocytic leukemia: strategies to define high-risk patients. Ann Hematol. 2016;95:673-80.
52. Montesinos P, Rayon C, Vellenga E, et al. Clinical significance of CD56 expression in patients with acute promyelocytic leukemia treated with all-trans retinoic acid and anthracycline-based regimens. Blood. 2011;117:1799-805.

53. Di Bona E, Avvisati G, Castaman G, et al. Early haemorrhagic morbidity and mortality during remission induction with or without all-trans retinoic acid in acute promyelocytic leukaemia. Br J Haematol. 2000;108:689-95.

\section{Affiliations}

\section{Sergio Matarraz ${ }^{1,2} \cdot$ Pilar Leoz $^{1,2} \cdot$ Carlos Fernández $^{1,2} \cdot$ Enrique Colado $^{3} \cdot$ María Carmen Chillón $^{2,4}$. María Belén Vidriales ${ }^{2,4} \cdot$ Marcos González $^{2,4}$ - Daniel Rivera ${ }^{2,4} \cdot$ Carlos Salvador Osuna $^{5}$. Teresa Caballero-Velázquez ${ }^{2,6}$ • Vincent Van Der Velden ${ }^{7}$ - Mojca Jongen-Lavrencic ${ }^{8}$ - Oliver Gutiérrez ${ }^{9}$. Ana Yeguas Bermejo ${ }^{10}$ - Luis García Alonso ${ }^{10}$ - Monique Bourgeois García ${ }^{11}$. Cristina De Ramón Sánchez ${ }^{11}$. Gloria García-Donas ${ }^{12}$ - Aránzazu García Mateo ${ }^{13}$ - Isabel Recio ${ }^{14}$ - Javier Sánchez-Real ${ }^{15}$. Andrea Mayado ${ }^{1,2}$. María Laura Gutiérrez ${ }^{1,2} \cdot$ Paloma Bárcena ${ }^{1,2}$. Susana Barrena ${ }^{1,2} \cdot$ Antonio López $^{1,2} \cdot$ Jacques Van Dongen $^{16}$. Alberto Orfao ${ }^{1,2}$}

1 Cytometry Service (NUCLEUS), Department of Medicine, IBSAL and CIBERONC, Cancer Research Center (IBMCC, University of Salamanca-CSIC), Salamanca, Spain

2 Centro de Investigación Biomédica en Red de Cáncer (CIBERONC), Madrid, Spain

3 Hematology and Laboratory Medicine Department and Instituto Universitario de Oncología del Principado de Asturias (IUOPA), Central University Hospital of Asturias, Oviedo, Spain

4 Hematology Service, University Hospital of Salamanca, IBSAL and CIBERONC, Salamanca, Spain

5 Hematology Service, Miguel Servet Hospital, Zaragoza, Spain

6 Hematology Service, Virgen del Rocío Hospital, Sevilla, Spain

7 Department of Immunology, Erasmus MC, University Medical Center Rotterdam, Rotterdam, The Netherlands
8 Department of Hematology, Erasmus MC, University Medical Center Rotterdam, Rotterdam, The Netherlands

9 Hematology Service, Río Hortega Hospital, Valladolid, Spain

10 Hematology Service, University Hospital of Getafe, Madrid, Spain

11 Hematology Service, University Hospital, Valladolid, Spain

12 Hematology Service, Juan Ramón Jiménez Hospital, Huelva, Spain

13 Hematology Service, Segovia General Hospital, Segovia, Spain

14 Hematology Service, Nuestra Señora de Sonsoles Hospital, Avila, Spain

15 Hematology Service, León Hospital, León, Spain

16 Department of Immunohematology and Blood Transfusion, Leiden University Medical Center, Leiden, The Netherlands 